\title{
Reactions of Polycyclic Alkylaromatics: 5. Pyrolysis of Methylanthracenes
}

\author{
C. Michael Smith and Phillip E. Savage \\ Dept. of Chemical Engineering, University of Michigan, Ann Arbor, MI 48109
}

\begin{abstract}
1-, 2- and 9-methylanthracene were pyrolyzed neat at temperatures between 350 and $450^{\circ} \mathrm{C}$ for batch holding times up to $300 \mathrm{~min}$. The pyrolysis proceeded through three parallel primary reaction pathways: one led to anthracene via demethylation; the second to dimethylanthracenes through methyl addition; and the third to methyl9,10-dihydroanthracenes through hydrogenation. The relative importance of these three paths varied for different methylanthracene isomers. The presence of these primary pathways can be rationalized in terms of recently elucidated hydrogentransfer mechanisms and other aspects of the developing free-radical chemistry of polycyclic alkylarenes. The demethylation rate at $400^{\circ} \mathrm{C}$ for the methylanthracenes and seven other methylarenes was correlated with Dewar reactivity numbers, which provide a measure of the localization energy, as In rate (arene yield $/ \mathrm{min})=3.7-7.1$ $N_{t s}$, where $N_{t s}$ is the Dewar reactivity number for the peripheral aromatic carbon atom bearing the methyl substituent. This correlation may be useful in molecularbased reaction models for the conversion of heavy hydrocarbon resources such as coals and heavy oils.
\end{abstract}

\section{Introduction}

Advances in analytical chemistry and computer technology have given chemical engineers the opportunity to take a molecular perspective on reactions involving complex materials. A good example is the conversion and upgrading of hydrocarbon resources such as coals and heavy crude oils. Improved analytical techniques have provided detailed structural information about these materials, and integrating this knowledge of the structure with an understanding of the reaction fundamentals for the different structural elements has led to the development of molecular-based reaction models (Savage and Klein, 1989; Gray, 1990; Neurock et al., 1989, 1990; Quann and Jaffe, 1992; Shinn, 1992).

Central to the successful implementation of this modeling approach is a knowledge of the reaction pathways and kinetics for the different structural elements. This knowledge can, in principle, be obtained from experimental and computational studies of model compounds that mimic the key structural features of the complex material.

Alkylaromatic moieties are important structural features of coals (Solum et al., 1989; Shinn, 1984; Berkowitz, 1988) and heavy oils and residua (Ali et al., 1990; Speight, 1988, 1989; Waller et al., 1989). This realization has prompted several pyrolysis studies of long-chain $n$-alkylbenzenes (for example, Savage and Klein, 1987a,b; Savage and Korotney, 1990; Mushrush and Hazlett, 1984; Blouri et al., 1985; Freund and Olmstead, 1989) and 1-dodecylpyrene (Savage et al., 1989). The pyrolysis network for this polycyclic compound differed from that of its single-ring analog by the presence of a major primary pathway that involved cleavage of the strong aryl-alkyl C-C bond. Such a path was unimportant for alkylbenzene pyrolysis. This striking difference in the reaction pathways for alkylbenzenes and alkylpyrenes motivated several additional studies with $n$-alkylpyrenes (Smith and Savage, 1991a,b; Freund et al., 1991; McMillen et al., 1992) and other long-chain polycyclic $n$-alkylarenes (Smith and Savage, 1991c). Further, the hydrogenolysis of the aryl-alkyl $\mathrm{C}-\mathrm{C}$ bond is not a reaction exclusive to long-chain polycyclics, for even methyl groups in polycyclic alkylarenes such as 9,10-dimethylanthracene (Virk and Vlastnik, 1992; Pope, 1987) and 1-methylpyrene (Smith and Savage, 1992) undergo hydrogenolytic cleavage during pyrolysis.

The recent experimental observations noted above demon- 
strate that the aryl-alkyl C-C bond in certain polycyclic structures is susceptible to facile thermal cleavage at temperatures around $400^{\circ} \mathrm{C}$. Accordingly, molecular-based reaction models for coals and heavy oils need to include this hydrogenolytic thermal reaction pathway to be complete.

The relevance of the reactions of polycyclic alkylaromatics to a number of different heavy hydrocarbon resources and the limited amount of information available on the reaction fundamentals for polycyclic methylarenes motivated this research. This article discusses the thermal reaction pathways, kinetics, and mechanisms for 1-, 2- and 9-methylanthracenes. Including the three different methylanthracene isomers in this investigation allowed us to explore the effect of the position on which the methyl substituent resides on the pyrolysis pathways and kinetics. Nearly all previous pyrolyses of polycyclic $n$-alkylarenes (for example, Savage et al., 1989; Smith and Savage, 1991a-c, 1992; Virk and Vlastnik, 1992; Pope, 1987; Freund et al., 1991) have involved compounds in which the substituent was located on the most reactive peripheral aromatic carbon atom.

\section{Experimental Studies}

1-, 2- and 9-methylanthracence were pyrolyzed at temperatures between 350 and $450^{\circ} \mathrm{C}$ in stainless steel, tubing bomb batch microreactors that have been described previously (Smith and Savage, 1992). All of the methylanthracenes and other methylarenes used in this investigation were commercially available and used as received. Background experiments showed that the reactor material did not affect the products formed from $n$-alkylarene pyrolyses (Savage et al., 1989). We also tested for the possibility of catalytic activity by adding stainless steel filings to the reactor and then conducting alkylarene pyrolysis reactions. These experiments resulted in no statistical evidence for disputing the hypothesis that the added stainless steel had no effect on the reactant conversion or product selectivities (Smith, 1992).

The reactors were typically loaded with approximately 10 $\mathrm{mg}$ of the model compound and $10 \mathrm{mg}$ of a demonstrably inert internal standard, each weighed to within $\pm 0.1 \mathrm{mg}$, and then sealed in a nitrogen-filled glove box. After being loaded and closed, the reactors were placed in an isothermal, fluidized sand bath set at the desired pyrolysis temperature. Upon reaching the desired holding time, the reactors were removed from the sand bath, and the reaction was quenched by immersing the reactors in room-temperature water. The reactors were then opened and their contents recovered by repeated extraction with benzene.

The reaction products were analyzed by gas chromatography using both flame ionization and mass spectrometric detectors. Product identification was accomplished by comparing retention times with those of authentic standards and inspecting mass spectra. Product molar yields, calculated as the number of moles of product formed divided by the number of moles of the reactant initially loaded into the reactor, were obtained from the chromatographic analysis using experimentally determined detector response factors. We estimate the uncertainty in these molar yields to be about $\pm 20 \%$ of the reported value. Additional details about the experimental procedure and analytical protocol have been reported previously (Smith and Savage, 1991a-c, 1992).

\section{Results}

Tables 1-3 list the results of the neat pyrolysis experiments with the three different methylanthracenes used in this study. These results are presented as the molar yields of the reactant and products formed at different temperatures and holding times. The molar yield of the reactant exceeding $100 \%$ in a few instances is a manifestation of the uncertainty in the experimental data.

In addition to the products listed in Tables 1-3, gaseous and benzene-insoluble products were also formed. The very small amount of model compound used in the experiments prevented the analysis of these products. The benzene-insoluble products most likely comprised methylanthracene oligomers (Virk and Vlastnik, 1992). The inability to quantify the yields of these products prevented complete closure of the mass balance.

1-Methylanthracene was pyrolyzed at 400,425 and $450^{\circ} \mathrm{C}$, and Table 1 summarizes the experimental results. The major products at short reaction times were anthracene, 1-methyl9,10-dihydroanthracene, and five different dimethylanthra-

\section{Table 1. Molar Yields (\%) from 1-Methylanthracene} Pyrolysis

\begin{tabular}{lccccc}
\hline & \multicolumn{5}{c}{ Time (min) at $400^{\circ} \mathrm{C}$} \\
\cline { 2 - 6 } Product & 60 & 150 & 200 & 240 & 300 \\
\hline 1-Methylanthracene & 75 & 56 & 76 & 47 & 37 \\
Anthracene & 2.9 & 9.3 & 4.8 & 10 & 17 \\
9,10-Dihydroanthracene & - & 0.6 & 0.2 & 0.7 & 1.5 \\
1,2,3,4-Tetrahydroanthracene & - & 0.2 & - & 0.3 & 0.8 \\
Dimethylanthracene I & 0.3 & 0.9 & 0.5 & 1.0 & 1.2 \\
Dimethylanthracene II & 0.3 & 1.1 & 0.5 & 1.2 & 1.8 \\
Dimethylanthracene III & 0.7 & 2.0 & 1.1 & 2.2 & 2.6 \\
Dimethylanthracene IV & 0.1 & 0.3 & 0.1 & 0.2 & 0.3 \\
Dimethylanthracene V & 1.0 & 1.3 & 1.4 & 1.0 & 0.7 \\
1-Methyl-9,10-dihydroanthracene & 2.3 & 3.4 & 2.7 & 2.9 & 3.3 \\
9-Methylanthracene & - & 0.5 & 0.1 & 0.5 & 0.9 \\
\hline
\end{tabular}

\begin{tabular}{lcccccccc}
\hline & \multicolumn{6}{c}{ Time (min) } & at & $425^{\circ} \mathrm{C}$ \\
\cline { 2 - 8 } Product & 30 & 60 & 90 & 120 & 150 & 240 & 300 \\
\hline 1-Methylanthracene & 81 & 66 & 35 & 33 & 22 & 16 & 9.5 \\
Anthracene & 4.0 & 11 & 19 & 16 & 24 & 25 & 27 \\
9,10-Dihydroanthracene & - & 0.5 & 1.7 & 1.2 & 2.8 & 2.5 & 3.5 \\
1,2,3,4-Tetrahydroanthracene & - & 0.1 & 0.7 & 0.5 & 1.4 & 1.6 & 2.2 \\
Dimethylanthracene I & 0.4 & 1.0 & 1.2 & 1.2 & 1.0 & 0.7 & 0.5 \\
Dimethylanthracene II & 0.4 & 1.2 & 2.0 & 1.7 & 2.1 & 1.6 & 1.3 \\
Dimethylanthracene III & 0.9 & 2.3 & 2.7 & 2.5 & 2.1 & 1.1 & 0.7 \\
Dimethylanthracene IV & 0.1 & 0.2 & 0.3 & 0.3 & 0.3 & - & - \\
Dimethylanthracene V & 1.3 & 1.5 & 6.8 & 0.7 & 0.4 & - & - \\
1-Methyl-9,10-dihydroanthracene & 2.0 & 2.9 & 2.7 & 2.3 & 2.2 & 2.2 & 1.4 \\
9-Methylanthracene & - & 0.6 & 0.8 & 0.8 & 0.9 & - & 0.3 \\
\hline
\end{tabular}

Time $(\min )$ at $450^{\circ} \mathrm{C}$

$\begin{array}{llllllll}\text { Product } & 30 & 60 & 90 & 120 & 150 & 240 & 300\end{array}$

1-Methylanthracene

Anthracene

9,10-Dihydroanthracene

1,2,3,4-Tetrahydroanthracene

Dimethylanthracene I

Dimethylanthracene II

Dimethylanthracene III

Dimethylanthracene IV

Dimethylanthracene $\mathrm{V}$

$\begin{array}{lllllll}43 & 30 & 21 & 9.5 & 8.2 & 4.4 & 2.7\end{array}$

$\begin{array}{lllllll}15 & 22 & 26 & 31 & 29 & 26 & 23\end{array}$

$\begin{array}{lllllll}0.9 & 1.7 & 2.6 & 3.4 & 3.2 & 3.5 & 3.4\end{array}$

$\begin{array}{lllllll}0.2 & 0.6 & 1.1 & 1.7 & 1.6 & 2.0 & 1.7\end{array}$

$\begin{array}{lllllll}1.1 & 1.2 & 1.0 & 0.6 & 0.3 & 0.2 & -\end{array}$

$\begin{array}{lllllll}1.5 & 2.1 & 2.1 & 1.4 & 0.9 & 0.4 & -\end{array}$

$2.52 .72 .0---$

$\begin{array}{llllll}0.4 & 0.4 & 0.4 & 0.3 & - & -\end{array}$

$\begin{array}{llllll}0.9 & 0.5 & 2.7 & - & - & -\end{array}$

$\begin{array}{llllllll}\text { 1-Methyl-9,10-dihydroanthracene } & 2.3 & 2.1 & 1.9 & 1.4 & 1.1 & 0.8 & 0.1\end{array}$

9-Methylanthracene 
Table 2. Molar Yields (\%) from 2-Methylanthracene Pyrolysis

\begin{tabular}{lccccccccc}
\hline & \multicolumn{6}{c}{ Time (min) at } & $400^{\circ} \mathrm{C}$ \\
\cline { 2 - 8 } Product & 15 & 45 & 60 & 75 & 90 & 105 & 120 & 130 \\
\hline 2-Methylanthracene & 101 & 86 & 95 & 132 & 88 & 81 & 82 & 67 \\
Anthracene & 0.1 & 0.2 & 0.2 & 0.6 & 0.7 & 0.8 & 1.0 & 1.2 \\
Dimethylanthracene & 0.1 & 0.4 & 0.4 & 0.8 & 0.7 & 0.7 & 0.9 & 0.8 \\
Dimethylanthracene & 0.1 & 0.2 & 0.1 & 0.2 & 0.1 & 0.2 & 0.1 & - \\
Dimethylanthracene & 0.2 & 0.4 & 0.4 & 0.7 & 0.4 & 0.4 & 0.5 & 0.4 \\
2-Methyl-9,10-dihydroanthracene & 1.7 & 2.6 & 2.5 & 4.2 & 3.4 & 3.6 & 3.8 & 3.5
\end{tabular}

\begin{tabular}{lllllllllll}
\hline & \multicolumn{6}{c}{ Time $(\min )$} & at & $425^{\circ} \mathrm{C}$ \\
\cline { 2 - 8 } Product & 30 & 45 & 60 & 75 & 90 & 110 & 150 & 200 \\
\hline 2-Methylanthracene & 82 & 79 & 70 & 76 & 77 & 68 & 67 & 54 \\
Anthracene & 2.5 & 3.8 & 4.3 & 4.8 & 2.3 & 3.3 & 6.7 & 8.9 \\
Dimethylanthracene & 0.7 & 1.7 & 2.1 & 2.4 & - & - & 0.8 & 1.2 \\
Dimethylanthracene & 0.1 & 0.2 & - & 0.3 & 1.4 & 1.7 & 2.9 & 3.3 \\
Dimethylanthracene & 0.4 & 1.1 & 1.4 & 1.4 & - & - & 1.7 & 1.4 \\
2-Methyl-9,10-dihydroanthracene & 1.3 & 3.4 & 4.7 & 5.7 & 3.8 & 4.5 & 5.9 & 4.7 \\
\hline
\end{tabular}

Time (min) at $450^{\circ} \mathrm{C}$

Product

$\begin{array}{lllllll}60 & 75 & 90 & 105 & 120 & 145 & 160200\end{array}$

2-Methylanthracene

Anthracene

Dimethylanthracene

Dimethylanthracene

Dimethylanthracene

2-Methyl-9, 10-dihydroanthracene $\begin{array}{lllllllll}4.9 & 5.8 & 5.3 & 5.7 & 6.8 & 5.1 & 4.7 & -\end{array}$

cene isomers. We were not able to determine the positions of the methyl groups in these last compounds, so we simply refer to these isomers as numbers $\mathrm{I}$ through $\mathrm{V}$. At longer reaction times, 9,10-dihydroanthracene, 1,2,3,4-tetrahydroanthracene, and 9-methylanthracene appeared as reaction products.

Anthracene was the most abundant product at all reaction conditions, and its yield typically increased with holding time. The highest anthracene yield was $31 \%$, which was obtained from pyrolysis at $450^{\circ} \mathrm{C}$ for $120 \mathrm{~min}$. At conversions less than $50 \%$, 1-methyl-9,10-dihydroanthracene was the second most abundant product, but at higher conversions its yield was low. For example, at $425^{\circ} \mathrm{C}$ the yield of 1-methyl-9,10-dihydroanthracene reached a maximum value of $2.9 \%$ at $60 \mathrm{~min}$, but it decreased to $1.4 \%$ by $300 \mathrm{~min}$. A maximum was also present in the temporal variations of each of the dimethylanthracene isomers and 9-methylanthracene. Such behavior was not apparent in the data for 9,10-dihydroanthracene and 1,2,3,4tetrahydroanthracene. Rather, the molar yields of these products were very low at short holding times, but they increased steadily with time. Only anthracene had a higher yield than these partially hydrogenated arenes at conversions exceeding $90 \%$.

2-Methylanthracene was pyrolyzed at 400,425 and $450^{\circ} \mathrm{C}$, and Table 2 summarizes the experimental results. The major product at $400^{\circ} \mathrm{C}$ was 2 -methyl-9,10-dihydroanthracene, and less amounts of anthracene and dimethylanthracenes were formed. At 425 and $450^{\circ} \mathrm{C}$, however, anthracene joined 2methyl-9,10-dihydroanthracene as one of the most abundant products. This product slate is analogous to the products formed from 1-methylanthracene pyrolysis, but there were two fewer dimethylanthracene isomers and no partially hydrogenated anthracenes detected from 2-methylanthracene pyrolysis.
Table 3. Molar Yields (\%) from 9-Methylanthracene Pyrolysis

\begin{tabular}{lcccccc}
\hline & \multicolumn{6}{c}{ Time (min) at $350^{\circ} \mathrm{C}$} \\
\cline { 2 - 8 } Product & 15 & 30 & 60 & 100 & 120 & 180 \\
\hline 9-Methylanthracene & 94 & 122 & 116 & 106 & 57 & 129 \\
Anthracene & 7.2 & 1.9 & 2.9 & 3.4 & 2.4 & 4.6 \\
9,10-Dihydroanthracene & 1.0 & - & 0.2 & 0.6 & 0.4 & 0.2 \\
Dimethylanthracene & 0.7 & - & - & 0.4 & - & 0.5 \\
Dimethylanthracene & 2.5 & 0.7 & 1.1 & 1.1 & 0.9 & 1.7 \\
\hline
\end{tabular}

\begin{tabular}{lcccccc}
\hline & \multicolumn{9}{c}{ Time (min) at $375^{\circ} \mathrm{C}$} \\
\cline { 2 - 7 } Product & 15 & 30 & 45 & 60 & 75 & 90 \\
\hline 9-Methylanthracene & 84 & 78 & 78 & 62 & 53 & 40 \\
Anthracene & 6.4 & 12 & 15 & 21 & 21 & 21 \\
9,10-Dihydroanthracene & 0.5 & 0.8 & 1.1 & 1.2 & 1.2 & 1.1 \\
Dimethylanthracene & 0.8 & 1.4 & 2.0 & 2.3 & 2.4 & 1.9 \\
Dimethylanthracene & 0.7 & 2.6 & 2.5 & 2.4 & 2.0 & 0.8 \\
\hline
\end{tabular}

\begin{tabular}{lcccccc}
\hline & \multicolumn{6}{c}{ Time (min) at $400^{\circ} \mathrm{C}$} \\
\cline { 2 - 7 } Product & 15 & 30 & 45 & 60 & 75 & 90 \\
\hline 9-Methylanthracene & 53 & 35 & 31 & 18 & 15 & 15 \\
Anthracene & 9.0 & 18 & 35 & 33 & 39 & 42 \\
9,10-Dihydroanthracene & 2.6 & 5.1 & 2.2 & 2.3 & 2.2 & 1.1 \\
Dimethylanthracene & 1.6 & 2.5 & 2.4 & 1.4 & 0.9 & 1.0 \\
Dimethylanthracene & 1.0 & 1.4 & 2.5 & 1.9 & 1.6 & 1.9 \\
\hline
\end{tabular}

9-Methylanthracene was pyrolyzed at 350,375 and $400^{\circ} \mathrm{C}$, and Table 3 provides the results. The major products were anthracene and a dimethylanthracene at $350^{\circ} \mathrm{C}$. 9,10-Dihydroanthracene became an abundant product at the higher temperatures.

Comparing the data in Tables 1-3 for the three different methylanthracenes shows that the order of reactivity was 9 . methylanthracene $>1$-methylanthracene $>2$-methylanthracene. Further, all of the methylanthracenes generally formed the same three types of products at low conversions; anthracene, a methyl-9,10-dihydroanthracene, and dimethylanthracenes. The relative amounts of these compounds differed for the different methylanthracenes pyrolyzed. In a subsequent section, we will consider this sensitivity of the pyrolysis kinetics and pathways to the location of the methyl substituent in more detail.

\section{Pyrolysis Pathways}

The data provided in the previous section facilitate the development of a pyrolysis network for methylanthracenes. An initial step in reaction pathway analysis determines the order of appearance of each of the reaction products. This task can be readily accomplished by employing the Delplot methodology (Bhore et al., 1990), which in its simplest form involves inspection of plots of product selectivities as a function of reactant conversion. These selectivities are calculated as the molar yield divided by the conversion. Primary products (those that arise directly from the reactant) possess positive intercepts on these Delplots, whereas products that appear later in the reaction network possess intercepts equal to zero. Thus, an examination of the Delplot intercepts provides rapid discrimination between primary and higher order products.

Figure 1 displays the Delplots for 1-methylanthracene py- 


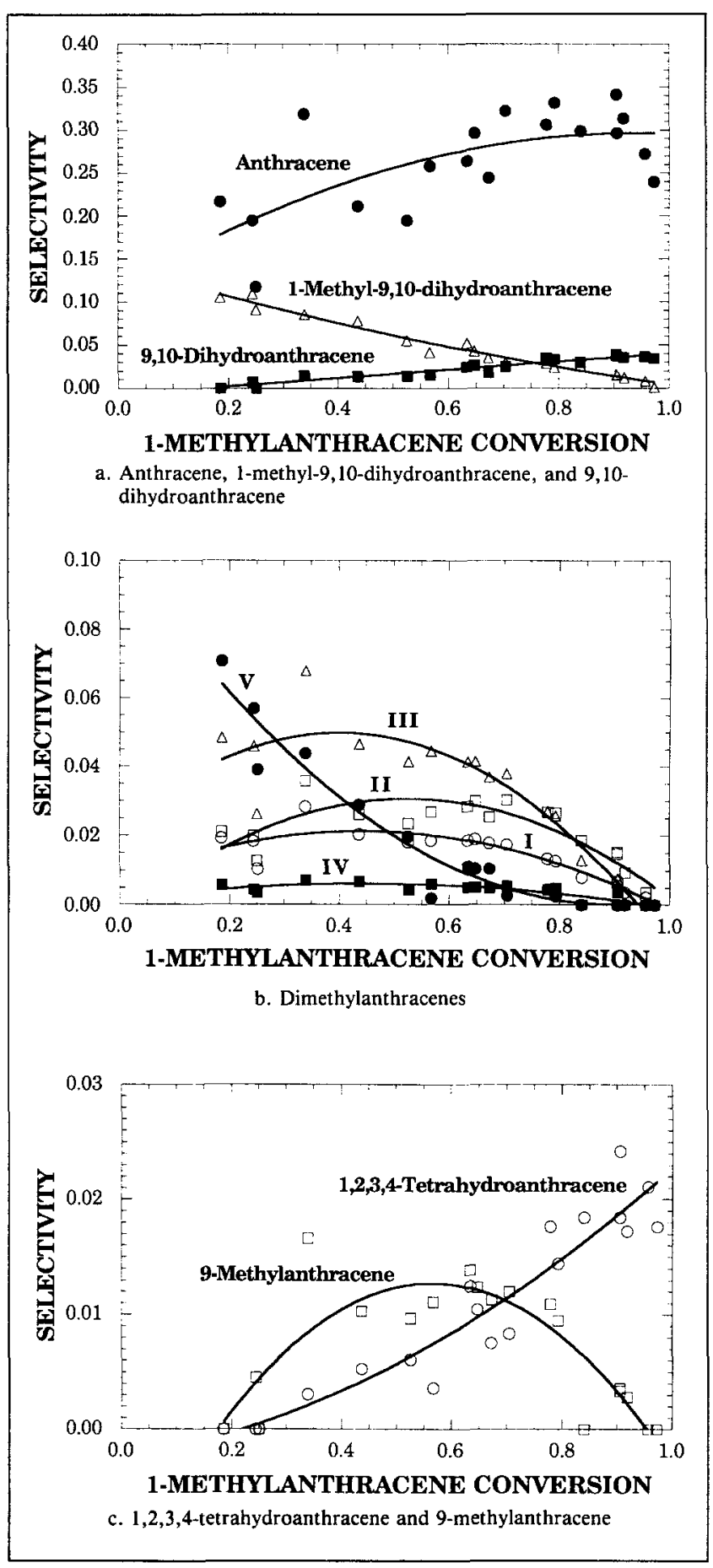

Figure 1. Delplots for 1-methylanthracene pyrolysis.

rolysis. The discrete points are the experimental values, and the curves are second-order polynomial fits provided to convey the trends in the data. Figure 1a shows that the initial selectivities to anthracene and 1-methyl-9,10-dihydroanthracene were both positive, which identify these compounds as primary products. The initial selectivity for 9,10-dihydroanthracene was zero, however, which identifies this compound as a nonprimary product. Figure 1b shows that dimethylanthracene isomers III and $\mathrm{V}$ exhibit nonzero initial selectivities, so these too are primary products. Determining the Delplot intercepts for isomers I, II and IV is not as straightforward; however, because the selectivities are low, the data are more scattered, and there are few low conversion points. Thus, the data for isomers I, II and IV are not conclusive, and these dimethylanthracenes could be either primary or higher-order products. Finally, the zero intercepts in Figure 1c show that 1,2,3,4-tetrahydroanthracene and 9-methylanthracene were not primary products.

Additional insight into the reaction pathways can be obtained from the variation of the product selectivities with conversion. For example, the 1-methyl-9,10-dihydroanthracene selectivity decreased with conversion. This behavior is consistent with the participation of this product in secondary decomposition reactions. In this light, it is interesting to note that the selectivity of 9,10-dihydroanthracene, a potential decomposition product, increased with conversion. The trends in the selectivity data for the dimethylanthracenes in Figure $1 \mathrm{~b}$ are consistent with each of these isomers having undergone secondary reactions.

To summarize, the Delplots in Figure 1 are consistent with 1-methylanthracene forming anthracene, 1-methyl-9,10-dihydroanthracene, and dimethylanthracenes in primary reaction paths. Higher-order reaction paths are responsible for the formation of 9,10-dihydroanthracene, 1,2,3,4-tetrahydroanthracene, 9-methylanthracene, and perhaps some dimethylanthracenes.

A similar reaction pathway analysis for the products from 2-methylanthracene pyrolysis led to the identification of anthracene, dimethylanthracenes, and 2-methyl-9,10-dihydroanthracene as primary products. Likewise, anthracene and dimethylanthracenes were primary products from 9-methylanthracene pyrolysis, whereas 9,10-dihydroanthracene was a secondary or higher order product.

The foregoing assignment of products as primary and secondary can be summarized by the reaction network in Figure 2. Here, we show primary reactions for each of the methylanthracenes leading to anthracene by demethylation and to dimethylanthracenes by methyl addition. The 1- and 2-methylanthracene isomers can undergo hydrogenation to form methyl-9,10-dihydroanthracenes. This pathway is not important for 9-methylanthracene, and we will discuss this observation further in a subsequent section dealing with reaction mechanisms. The methyl-9,10-dihydroanthracenes can be demethylated to yield 9,10-dihydroanthracene, but this product can also be formed via hydrogenation of anthracene.

Figure 2 shows that a path for the production of 9-methylanthracene from 1-methylanthracene involves an initial demethylation to form anthracene followed by methyl addition at the 9 position. Furthermore, addition of a second methyl group would account for the formation of dimethylanthracenes as nonprimary products.

The reaction network in Figure 2, which summarizes the pyrolysis pathways for methylanthracenes, comprises three parallel primary steps. These reversible steps involve methyl addition, hydrogenation, and demethylation. Thus, the pathways in Figure 2 can be generalized to develop a reaction network for the pyrolysis of other polycyclic methylarenes. This reaction network is shown in Figure 3, and it is consistent with our previous results from 1-methylpyrene pyrolysis (Smith and Savage, 1992) and with the pathways (Virk and Vlastnik, 1992) presented for 9,10-dimethylanthracene pyrolysis. Note 


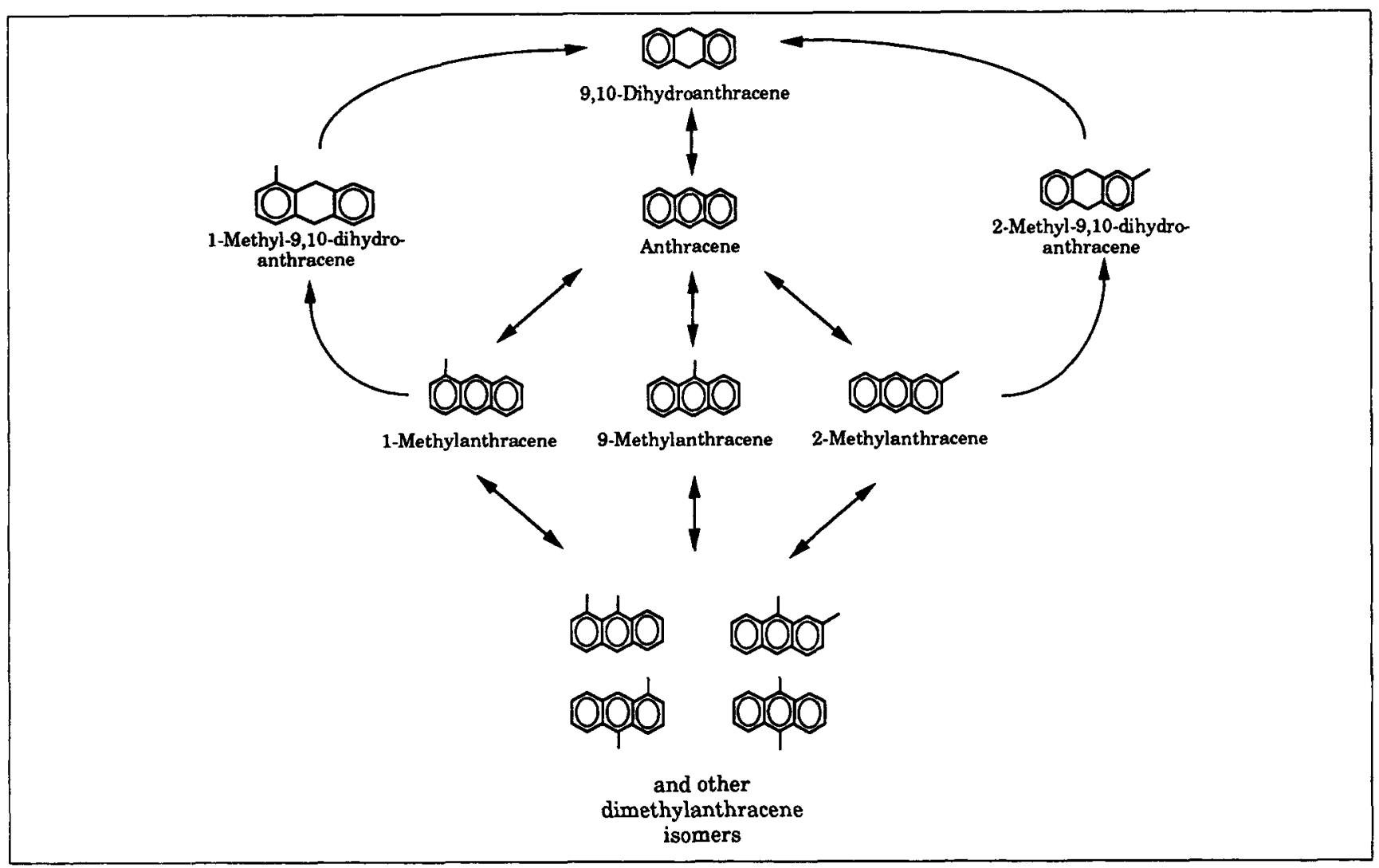

Figure 2. Pyrolysis network for methylanthracenes.

that Figures 2 and 3 describe the formation of the major products identified and quantified in this investigation. These reaction networks do not include the formation of the benzeneinsoluble products, because the identities and yields of these products could not be determined. The formation of these products, presumably anthracene oligomers, appeared to increase with the severity of the reaction conditions.

\section{Reaction Mechanisms}

The experimental results and reaction pathways discussed above can be rationalized in the light of recent work on the reaction mechanisms for polycyclic aromatics. Figure 4 shows the key elementary reaction steps for methylanthracenes; however, it does not provide a complete mechanism for methylanthracene pyrolysis and simply highlights the key steps that lead to the major products. Virk and Vlastnik (1992), Pope (1987), Smith and Savage (1992), and Billmers et al. (1986, 1989) provide more detailed mechanistic information on the

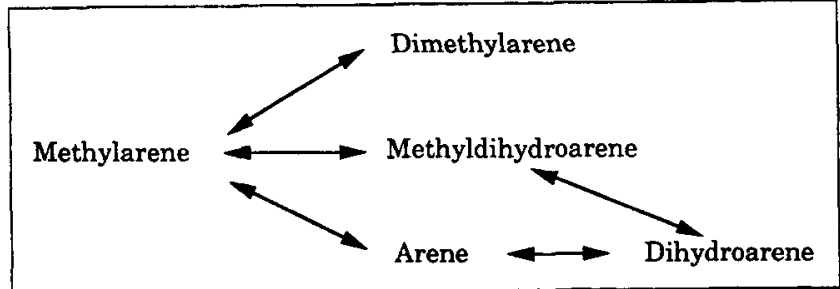

Figure 3. General pyrolysis network for polycyclic methylarenes.

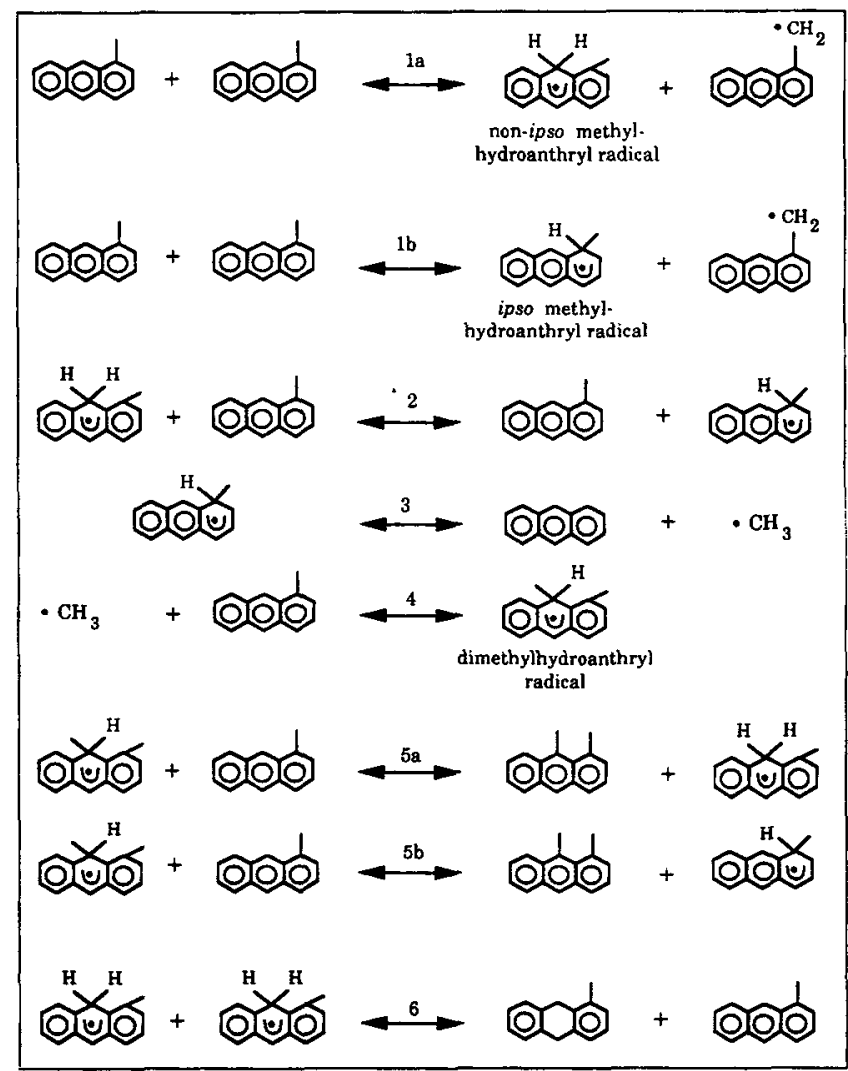

Figure 4. Key elementary reaction steps for methylan. thracene pyrolysis. 
thermal reactions of anthracenes and polycyclic methylarenes.

Initiation, steps $1 \mathrm{a}$ and $1 \mathrm{~b}$, occurs through the reverse of radical disproportionation (sometimes termed molecular disproportionation). These bimolecular steps transfer a benzylic hydrogen from one methylanthracene to a peripheral aromatic carbon atom on another methylanthracene. Hydrogen transfer to an unsubstituted position (step 1a) produces a nonipso methylhydroanthryl radical, whereas hydrogen transfer to the substituted position (step $1 \mathrm{~b}$ ) results in an ipso radical. The nonipso radicals can transfer hydrogen to methylanthracene via step 2 to form additional ipso methylhydroanthryl radicals. The ipso methylhydroanthryl radical can then eliminate a methyl radical and produce anthracene, one of the primary products, as shown in step 3. Step 4 shows a methyl radical adding to one of the peripheral carbons in methylanthracene to form one of the many possible dimethylhydroanthryl radicals. These radicals can subsequently transfer hydrogen to methylanthracene in step 5 to form dimethylanthracenes, which were primary products, and a methylhydroanthryl radical. The final primary product, methyl-9,10-dihydroanthracene, can form via step 6, a radical disproportionation step involving two nonipso methylhydroanthryl radicals.

Aside from accounting for the formation of the major primary products, the elementary steps in Figure 4 can also explain the absence of a methyl-substituted dihydroanthracene as a primary product from 9-methylanthracene pyrolysis. Formation of the most stable dihydroanthracene requires hydrogen addition to the 9 and 10 positions. Adding hydrogen to the 9 position in 9-methylanthracene, however, would form an ipso methylhydroanthryl radical, which would rapidly eliminate the methyl substituent via reaction 3 . Thus, the hydrogen transfer step required for producing a dihydroanthracene also facilitates cleavage of the aryl-alkyl $\mathrm{C}-\mathrm{C}$ bond and the loss of the methyl group in 9-methylanthracene.

\section{Hydrogenolysis Kinetics}

The data in Tables 1-3 showed that the reactivity of the three methylanthracenes differed. These different reaction rates must arise from some effect related to the position bearing the methyl substituent, because all other factors were identical for these three compounds. We recently showed (Smith and Savage, 1991c) that the effect of structure on the rate of dealkylation of long-chain polycyclic $n$-alkylarenes was related to the localization energy associated with the aromatic carbon atom bearing the aliphatic substituent. This localization energy is the difference in $\pi$-electron binding energy between the initial and transition states in an aromatic substitution reaction such as:

$$
Y+A r-X \rightarrow[Y-A r--X]^{\ddagger} \rightarrow A r-Y+X .
$$

For a family of reactions in which $X$ and $Y$ are constant, the activation energy will be determined by differences in the localization energies. Therefore, the rate constants for a family of aromatic substitution reactions should correlate linearly with the localization energy on semilogarithmic coordinates.

Dewar (1952) showed that the localization energies can be calculated from perturbation molecular orbital theory and conveniently expressed in terms of reactivity numbers. Other factors being equal, the smaller the Dewar reactivity number (or

\begin{tabular}{|c|c|c|}
\hline COMPOUND & STRUCTURE & DEWAR NUMBER \\
\hline 9-Methylanthracene & & 1.26 \\
\hline 7-Methylbenz(a)anthracene & & 1.35 \\
\hline 1-Methylpyrene & & 1.51 \\
\hline 7-Methylbenzo(a)pyrene & & 1.55 \\
\hline 1-Methylanthracene & & 1.57 \\
\hline 6-Methylchrysene & & 1.67 \\
\hline 1-Methylnaphthalene & & 1.81 \\
\hline 2-Methylanthracene & & 1.89 \\
\hline 2-Methylnaphthalene & & 2.12 \\
\hline 2-Methylphenanthrene & & 2.18 \\
\hline
\end{tabular}

Figure 5. Polycyclic methylarenes and the Dewar numbers of the substituted positions.

analogously the smaller the localization energy), the more rapidly should hydrogen addition and displacement of alkyl substituents occur. Our previous study (Smith and Savage, 1991c) of dealkylation in long-chain polycyclic alkylarenes was consistent with this expectation. We showed that the natural log of the initial rate of dealkylation for eight different long-chain $n$-alkylarenes correlated linearly with the Dewar number of the substituted position. In this section, we use these Dewar reactivity numbers to develop a structure-reactivity correlation for the demethylation of methylanthracenes and the other seven polycyclic methylarenes displayed in Figure 5.

Figure 6 shows a semilog plot of the initial rates of demethylation (arene yield $/ \mathrm{min}$ ) at $400^{\circ} \mathrm{C}$ for the three methylanthracenes and for the other seven methylarenes as a function of the Dewar number of the position bearing the methyl substituent $\left(N_{t s}\right)$. The demethylation rates for the methylanthracenes were calculated using the data at $400^{\circ} \mathrm{C}$ in Tables 1-3. The three points in Figure 6 for 1-methylpyrene demethylation were calculated from two sets of data reported by Smith (1992) and from one additional set of experiments performed more recently in our laboratory. These three points, which were determined at the same nominal reaction conditions but by different investigators over the course of nearly 18 months, indicate the amount of variability inherent in these initial demethylation rate measurements. Figure 6 shows the expected trend that low Dewar numbers are associated with high reactivities. Furthermore, the data tend to form a single linear 


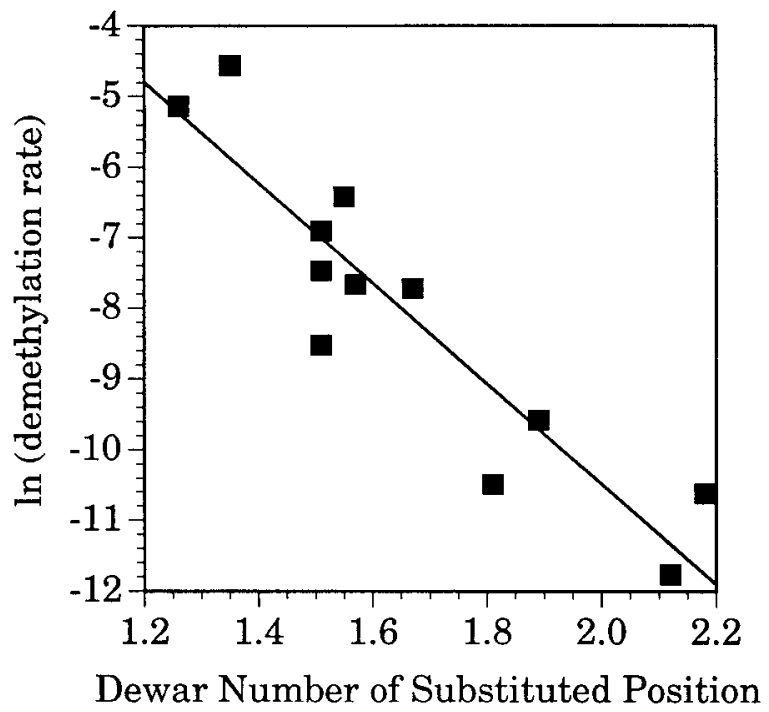

Figure 6. Correlation of the initial demethylation rate at $400^{\circ} \mathrm{C}$ with Dewar reactivity numbers for the methylarenes in Figure 5.

correlation, which is consistent with our previous data for the pyrolytic dealkylation of long-chain polycyclic $n$-alkylarenes (Smith and Savage, 1991c). The solid line in Figure 6 is the best linear fit to the data, and its equation is $\ln ($ rate $)=3.7-7.1$ $N_{t s}$.

Although Figure 6 does appear to exhibit the expected linear trend, there is clearly some scatter about the regressed line in Figure 6. Some of this scatter can be attributed to the uncertainty in the experimental measurements. Another reason that all of the data do not fall on the line, however, is that the dealkylation rate law may be complex, and the localization energy of the substituted position may not be the sole significant parameter. A consideration of the elementary steps in Figure 4 can assist in this mechanistic interpretation of the results in Figure 6.

Demethylation and formation of the unsubstituted arene occur via elimination of the methyl group from an ipso methylhydroaryl radical as shown in step 3 of Figure 4. This step is very rapid, so the rate of forming the ipso radical largely controls the rate of arene formation. Formation of the ipso radical requires that hydrogen be transferred from a donor to the substituted position. Accordingly, one could reasonably expect the nature of the hydrogen donor, in addition to the Dewar number for the substituted position, to influence the rates of these hydrogen transfer steps.

The two key hydrogen donors in Figure 4 are the methylarene itself (see step $\mathrm{lb}$ ) and nonipso hydroaryl radicals (see steps 2 and $5 \mathrm{~b}$ ). When the donor is the methylarene, a benzylic $\mathrm{C}-\mathrm{H}$ bond must be broken. The strength of this bond depends on the energetics of the substituted position. Therefore, the Dewar number of the substituted position should be the only parameter necessary to correlate the rate of this hydrogen transfer step. Consequently, if all hydrogen transfer were via these reverse radical disproportionation reactions, the dealkylation rates for all of the compounds in Figure 5 should fall on a single line in Figure 6. A failure of the data in Figure 6 to fall on a single line can then be attributed, in part, to reverse radical disproportionation not being the exclusive hydrogen transfer step. Such a scenario is fully consistent with our mechanistic modeling results for 1-methylpyrene pyrolysis (Smith and Savage, 1992), which showed that reverse radical disproportionation was but one of the important hydrogen transfer steps. The other important hydrogen donors in methylarene pyrolysis are nonipso hydroaryl radicals. The rates of these radical hydrogen transfer steps depend on the energetics of the particular nonipso position involved. Consequently, one could expect the Dewar numbers for nonipso positions to be important in correlating hydrogenolysis rates. Furthermore, the most stable nonipso hydroaryl radical (that is, the one formed by hydrogen addition to the unsubstituted position with the lowest Dewar number) is expected to be the dominant radical hydrogen donor and, hence, the most important in determining the hydrogenolysis rate. Therefore, the rate of hydrogen transfer (and subsequent dealkylation) by nonipso hydroaryl radicals can be reasonably expected to vary with the Dewar number for the most reactive unsubstituted position. To summarize, this discussion of the key hydrogen transfer steps leads us to speculate that the rate of demethylation of polycyclic methylarenes may generally depend on both the Dewar number of the substituted position and the most reactive unsubstituted position.

To test this hypothesis, we completed a multiple linear regression of the demethylation rate data using the Dewar reactivity numbers for the substituted position and for the most reactive unsubstituted position $\left(N_{t r}\right)$ as the independent variables. The resulting correlation was $\ln$ (rate) $=4.7-6.4 N_{t s}-1.4$ $N_{t r}$, but the fit of the rate data did not improve sufficiently to justify the inclusion of $N_{t r}$ as a second correlating parameter. The inability of the multiple linear regression to give a statistically significant dependence of the demethylation rate on $N_{t s}$ could be due, in part, to the uncertainty in the experimental rate data. Another reason could be that the linear model used here might not be consistent with the global hydrogenolysis rate law. Indeed, the linear model would be strictly correct, only if the rate constants for RRD and RHT steps appeared as products in the demethylation rate law. In this case, the influence of the localization energies on the apparent activation energy would be additive. If the rate law is more complex, there would be no reason to expect the influence of the localization energies to be additive. Further work on the kinetics and mechanisms of demethylation is required to determine the hydrogenolysis rate law.

The initial demethylation rate at $400^{\circ} \mathrm{C}$ for the ten polycyclic methylarenes in Figure 5 can be correlated reasonably well with the Dewar reactivity number for the substituted position, $N_{t s}$, as $\ln$ (rate) $=3.7-7.1 N_{t s}$. This equation can be used in molecular-based reaction models to account for thermal demethylation reactions in heavy hydrocarbon resources.

\section{Summary and Conclusions}

Methylanthracene pyrolysis proceeds through three parallel primary pathways that involve demethylation, methylation, and hydrogenation, respectively. The relative importance of the three pathways varied for the three different methylanthracenes. These differences in reactivity were explained by considering the influence of the localization energies associated with the different peripheral aromatic carbon atoms on the rates of the key elementary reaction steps. The initial rate of demethylation during methylarene pyrolysis was quantitatively 
correlated with the Dewar reactivity number for the substituted position. This structure-reactivity correlation provides a link between the location of a methyl group on a polycyclic aromatic nucleus and the susceptibility of the methyl group to hydrogenolytic displacement. As such, it may prove useful in molecular-based reaction models for heavy hydrocarbon resources.

\section{Acknowledgment}

Minda McNally, Scott Schneider, and Steve Sherman conducted the methylanthracene, 1-methylnaphthalene, and some of the 1-methylpyrene pyrolyses, respectively. This work was supported in part by the Link Foundation, the Shell Faculty Career Initiation Fund, and the National Science Foundation (CTS-8906859). Acknowledgment is also made to the donors of the Petroleum Research Fund (ACS-PRF 23744-AC4), administered by the ACS, for the partial support of this research.

\section{Literature Cited}

Ali, L. H., K. A. Al-Ghannam, and J. M. Al-Rawi, "Chemical Structure of Asphaltenes in Heavy Crudes Investigated by n.m.r.," Fuel, 69, 519 (1990).

Berkowitz, N., Polynuclear Aromatic Compounds, ACS Adv, Chem. Ser., No. 217, p. 217 (1988).

Bhore, N. A., M. T. Klein, and K. B. Bischoff, "The Delplot Technique: A New Method for Reaction Pathway Analysis," Ind. Eng. Chem. Res., 29, 313 (1990).

Billmers, R., L. L. Griffith, and S. E. Stein, "Hydrogen Transfer Between Anthracene Structures," J. Phys. Chem., 90, 517 (1986).

Billmers, R., R. L. Brown, and S. E. Stein, "Hydrogen Transfer Between 9,10-Dihydrophenanthrene and Anthracene," Int. J. Chem. Kinet., 21, 375 (1989).

Blouri, B., F. Hamdan, and D. Herault, "Mild Cracking of HighMolecular-Weight Hydrocarbons," Ind. Eng. Chem. Proc. Des. Dev., 24, 30 (1985).

Dewar, M. J. S., "Molecular Orbital Theory of Organic Chemistry VI. Aromatic Substitution and Addition," J. Amer. Chem. Soc., 74, 3357 (1952).

Freund, H., M. G. Matturro, W. N. Olmsted, R. P. Reynolds, and T. H. Upton, "Anomalous Side Chain Cleavage in Alkylaromatic Pyrolysis," Energy and Fuels, 5, 840 (1991).

Gray, M. R., "Lumped Kinetics of Structural Groups: Hydrotreating of Heavy Distillate," Ind. Eng. Chem. Res., 29, 505 (1990).

McMillen, D. F., J. A. Manion, and R. Malhotra, "Biaryl Formation as a Source of Hydrogen During Pyrolysis of Resid Structural Elements," ACS Div. Fuel Chem. Prep. 37, 1636 (1992).

Mushrush, G. W., and R. N. Hazlett, "Pyrolysis of Organic Compounds Containing Long Unbranched Alkyl Groups," Ind. Eng. Chem. Fundam., 23, 288 (1984).

Neurock, M., C. Libanati, A. Nigam, and M. T. Klein, "Monte Carlo Simulation of Complex Reaction Systems: Molecular Structure and
Reactivity in Modelling Heavy Oils," Chem. Eng. Sci., 45, 2083 (1990).

Neurock, M., C. Libanati, and M. T. Klein, "Modeling Asphaltene Reaction Pathways: Intrinsic Chemistry," AIChE Symp. Ser., 273(85), 7 (1989).

Pope, J. M., "The Kinetics and Mechanism of Thermal Hydrogen Transfer Reactions between Hydrocarbons," ScD Thesis, MIT (1987).

Quann, R. J., and S. B. Jaffe, "Structure-Oriented Lumping: Describing the Chemistry of Complex Hydrocarbon Mixtures," Ind. Eng. Chem. Res., 31, 2483 (1992).

Savage, P. E., and M. T. Klein, "Asphaltene Reaction Pathways: 2. Pyrolysis of $n$-Pentadecylbenzene," Ind. Eng. Chem. Res., 26, 488 (1987a).

Savage, P. E., and M. T. Klein, "Discrimination Between Molecular and Free-Radical Models of 1-Phenyldodecane Pyrolysis," Ind. Eng. Chem. Res., 26, 374 (1987b).

Savage, P. E., and M. T. Klein, "Asphaltene Reaction Pathways: 5. Chemical and Mathematical Modeling," Chem. Eng. Sci., 44, 393 (1989).

Savage, P. E., and D. J. Korotney, "Pyrolysis Kinetics for LongChain n-Alkylbenzenes: Experimental and Mechanistic Modeling Results," Ind. Eng. Chem. Res., 29, 499 (1990).

Shinn, J. H., "Reactive Model of Coal Structure," Fuel, 63, 1186 (1984).

Shinn, J. H., "Molecular Model of Reforming Chemistry at Reformulated Gasoline Conditions," ACS Div. Fuel Chem. Prep., 37, 50 (1992).

Smith, C. M., "Reactions of Polycyclic Alkylaromatics: Pathways, Kinetics, and Mechanisms," PhD Diss., Univ. of Michigan (1992).

Smith, C. M., and P. E. Savage, "Reactions of Polycyclic Alkylaromatics: 1. Pathways, Kinetics, and Mechanisms for 1-Dodecylpyrene Pyrolysis," Ind. Eng. Chem. Res., 30, 331 (1991a).

Smith, C. M., and P. E. Savage, "Reactions of Polycyclic Alkylaromatics: 2. Pyrolysis of 1,3-Diarylpropanes," Energy and Fuels, 5, 146 (1991b).

Smith, C. M., and P. E. Savage, "Reactions of Polycyclic Alkylaromatics: 3. Structure and Reactivity," AIChE J. 37, 1613 (1991c).

Smith, C. M., and P. E. Savage, "Reactions of Polycyclic Alkylaromatics: 4. Hydrogenolysis Mechanisms in 1-Alkylpyrene Pyrolysis," Energy and Fuels, 6, 195 (1992).

Solum, M. S., R. J. Pugmire, and D. M. Grant, " ${ }^{13}$ C Solid State NMR of Argonne Premium Coals," Energy and Fuels, 3, 187 (1989).

Speight, J. G., Polynuclear Aromatic Compounds, ACS Adv. Chem. Ser., No. 217, p. 201 (1988).

Speight, J. G., "Latest Thoughts on the Molecular Nature of Petroleum Asphaltenes," ACS Div. Petr. Chern. Prep., 34, 321 (1989).

Virk, P. S., and V. J. Vlastnik, "Pathways for Thermolysis of 9,10 Dimethylanthracene," ACS Div. Fuel Chem. Prep., 37, 947 (1992).

Waller, P. R., A. Williams, and K. D. Bartle, "The Structural Nature and Solubility of Residual Oil Fractions," Fuel, 68, 520 (1989).

Manuscript received Sept. 16, 1992, and revision received Jan. 25, 1993. 